$\xi=-1$

\title{
An Effective Software Reliability Estimation with Real-Valued Genetic Algorithm
}

\author{
Dr.G KrishnaMohan'1, B sowmya², KMohanvamsi ${ }^{3}$, K Sandeep ${ }^{4}$ \\ ${ }^{1,2,3,4}$ Dept. Of CSE, KoneruLakshamaiah Education Foundation, Vaddeswaram, Guntur Dist., AP-INDIA \\ *Corresponding author E-mail: gvlkm@kluniversity.in
}

\begin{abstract}
The implemented approach is powerful method for estimating reliability of the software parameters growing SRGM by utilizing an Algorithm which is known as RGA. The full form of RGA is Real-valued Genetic Algorithm. Parameters required for current SRGM, if we take an illustration, the Failures average number or identification rate of the failure utilizing the techniques which are numerical, estimation of the maximum probability or estimation of minimum square.RGA means the free form of SRGM parameter estimation limitations. Instead of these, this can be much adapted for optimizing domain continuously compared to the algorithm of the binary genetic. The operators of GA which is 2 real valued crossovers\& mutation of non-uniform interfaced for enhancing SRGM parameters estimation execution and accuracy enhancement. I led tests over eight datasets which are real valued to contrast implemented scheme \& techniques of the numerical \& another generic algorithm which are typical. The results describes that in estimation of SRGM parameters, the RGA is the most powerful compared to the others. So that we can trust the RGA which is the right solution for getting the efficient software quality with estimation of reliable accuracy.
\end{abstract}

Keywords: Softwarereliabilitygrowthmodel, Genetialgorithm, Goel-Okumoto model.

\section{Introduction}

The quality control of software prior to delivery is the most troublesome issue with the software industry. Therefore, there is a need for a precise and rapid reliability of software evaluation in a cycle which is software development. Models of SRGM software stated to assess the software reliability. For observing the software failure in control behaviour with 2 failures by SRGM. Failures time / how many times it fails. usually the parameters of SRGM is unknown \& have to evaluate according to the collected data. The famous 2 SRGM evaluation methods are estimation of maximum likelihood (MLE) \& estimation of least squares LSE. The LSE which is strategy of maths for gauging statistical model parameters which limited residuals square sum among the information which are anticipated as well as genuine. In any case, MLE is viewed as a superior measurable evaluator for vast example sizes, while LSE is appropriate for little and medium information. MLE along with LSE incorporate to likelihood hypothesis property \& factual investigation. Therefore, it can establish some limitations in the parameters of the SRGM, such as continuity, existence of derivatives. If SRGM is complex or fault data sample size is large, for 2 evaluation methods cannot be effective in finding the optimal solutions. Therefore, it is essential that SRGM have more effective and applicable approaches to parametric estimation. As the parameter estimation can be reviewed as part of the search for possible solutions, may be attractive for GA in the software reliability modelling process. Here, we implemented a algorithm of Real-valued genetic (RGA) for estimating the SRGM configuration.
As of now, the enthusiasm to apply met heuristics like the algorithm of genetic or the algorithm of particle swarm (PSO), for appraising the SRGM parameter expanded. Since studied strategies will the form of free strategies of numerical estimation imperatives, meta-heuristics used to estimate SRGM parameters. From the strategies, Darwin's regular hypothesis depend on GA, that expressed better people for the adjustment environment, opportunities more they have for nature living(Holland, 1975). GA gives an answer to for the Optimization in local issue with monotonous genetic operations performance for generating chromosomes.

Some relevant works utilizing GA strategies are here SRGM parameters estimation. First Minohara\&Tohma interconnected GA to the estimation of SRGM parameter. .They assessed HyperGeometric distribution software growth model (HGRM) the parameters utilizing a typical GA (CGA). Chao-Jung \& Huang implemented GA (MGA) Modified with a mechanism of reconstructing \& weighted bit mutation for evaluation SRGM parameters. The mechanism of reconstructing \& weighted bit mutation utilized for enhancement the GA execution for SRGM parameters estimation

These relevant works utilized the algorithm of Binary genetic (BGA). This BGA means strategy of GA which controls chromosomes for utilizing the operations of the binary genetic. Every chromosome is described with arrangement of genetic operations bit strings. Nonetheless, strategy isn't very much optimizing the continuous domain. As the GA genotype difference and phenotype, this can be extremely firm design efficient bit string with constant variables genetic operators such as SRGM parameters. If the operations of genetic depend over the bit manipulations, the every chromosome assessments will be done based on the real-valued computation in BGA. This needs extra procedures to encode and interpreting chromosomes among the strings of bit and real values. 
The extra procedures lead degradation performance in the estimation of parameter.

Here, we implemented an efficient way for dealing assess of SRGM parameters to use Real-valued GA (RGA). RGA describing for each one chromosome like a real values set $\&$ chromosomes will be straightforward in nature remodified with use of GA which is real-valued. 2 GA real-valued operations such as crossover heuristic \& mutation of non-uniform interfaced for SRGM parameter estimation exactness as well as performance.

For the implemented method, 8 real certifiable set of data as well as 5 SRGMs which are custom in nature. In this we can an output which is more reliable \& exact compared to typical systems.

\subsection{Goel-Okumoto Model (1979)}

This model was first presented by Goel and Okumoto and is based upon the following: Assumptions:

1. All software deficiencies are commonly autonomous from the purpose of failure see

2. The faults number detected at whatever the time in proportion to current number deficiencies found in the software. This means that the probability of real faults or defects, that is, is permanent.

3. *Isolated faults are eliminated from future test cases.

4. Each time the software fails, a software error causes it to be deleted immediately and no new errors are reported.

These assumptions lead below equation which is differential .In the equation case, a is presented during the test of the complete count common errors expected in software. Parameter $\mathrm{b}$ indicates failure rate of failure detection or failure, and (t) Failures which are expected over time. The previous solution $(t)$ for the equation, we obtain the following function of mean value

For example, the GO model equation is as follows:

$m(t)=a\left(1-e^{-b t}\right), \mathrm{a}>0, \mathrm{~b}>0$

In any case, these techniques doesn't suitable if SRGM mean value function may be non-direct (Minohara and Tohma, 1995). SRGM parameter estimation forces a few limitations requiring the coherence as well as derivatives presence of the function of modeling.

\section{GA Fundamental Concepts}

A population, make by GA is an setups arrangement named as chromosomes, evolve. The algorithm utilizes operators of three fundamentals: selection; crossover \& mutation (Fig. 1 describes). operator of the Selection operates new populace beginning with the present one, with the help of chromosomes empowering the have much grounded fitness. The administrator crossover operator utilizes the data presented two chromosomes, so as to construct another two. The mutation plays out an irregular change of a chromosome, keeping in mind the end goal to bring assorted variety. GA seen such as local search strategies using neighbourhoods two levels: neighbourhood of local, characterized with operator of the mutation operator as well as neighbourhood of the extensive, having to give population.

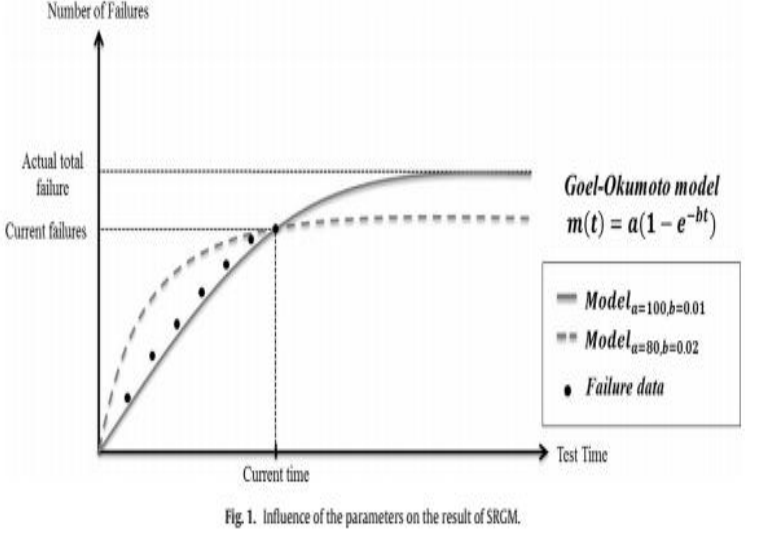

Fig1: Goel-Okumoto model Influence of parameter on the result of SRGM.

\subsection{Chromosomes Representation}

The GAs using primary issues is the individual's portrayal. This coding connected to optimization problem specifications. For the continuous variables functions optimization binary coding of classic does not adjust around. For esteems change of Indeed Gas for handling discrete variables with the help of small step . In frequent streamlining, the continuous variables representation with a completed alphabet that leads to difficulties.

\subsection{Population Structure}

Single population of GA produced usually domain D inside. GAs One difficulties are which regular converge much fast they make fast equal chromosomes. population, objective function local minima can be easily trapped it. This due to the population diversity premature in search. population another organization can be used to solve this at initial of algorithm. Entire idea follows one: divide population into subpopulations. several minima permits an exploration of function at equal time id the advantage of it, that allows for keeping the algorithm second step, only find the best point.

\section{Major Advantages}

GA has gotten impressive consideration viewing own potential like novel optimization method. 3 imperative central focuses there while applying GA to optimization problems:

\subsection{Adaptability}

GA does not have much mathematical necessity with respect to optimization issues. Because of the nature of evolutionary, GA scan to solutions with no respect for particular issue of inward workings. GA deal with whatever objective function sort and any requirements sort i.e. direct characterized or search space which are mixed.

\subsection{Robustness:}

The evolution operators utilization makes extremely successful GAwhile playing out a world hunt, If much customary heuristics functions local search. GA can be much efficient as well as robust of optimal solution locating as well as computational effort reduction compared to typical conventional heuristics. Proved. 


\subsection{Flexibility:}

The GA furnishes awesome adaptability for Hybridizing with the help of the Heuristics of Domain-Dependent for making a proficient usage to particular issue.

\section{Genetic Operators}

A Genetic Operator is an administrator utilized as a part of GA to keep up Diversity of the Genetic. Variety of Genetic need to process advancement. Operator of the Genetic utilized as a part of GA are practically equivalent for regular world: fittest survival, or Determination; Proliferation (Crossover, also known Recombination); \& Mutation. Assorted Variety of the Genetic, the biodiversity level, alludes for aggregate Genetic number qualities in the $n$ animal types genetic makeup . Recognized with the help of fluctuation of the genetic, that portrays genetic qualities inclination for differing. The populace genetic qualities scholastic field in corporated some speculations as well as hypotheses based on genetic diversity. The development neutral hypothesis recommends where diversity is neutral substitutions aggregation consequence .

$\mathrm{V}$ of the Diversifying means speculationwhich an animal types of two subpopulations of lives various conditions which is various alleles on specific locus. Id done sometimes, Take example, When species having a huge range with respect to inside people versatility . choice of the Recurrence subordinate means speculation which alleles turn out to be more typical, they turn in to the much helpless. At the point when GA continues, both the inquiry heading to ideal arrangement and the pursuit speed ought to be considered as vital components, to keep a harmony amongst investigation and abuse in look space. When all is said in done, the abuse of the collected data coming about because of GA look is finished by the choice instrument, while the investigation to new locales of the scan space is represented by hereditary administrators. The hereditary administrators imitate the procedure of heredity of qualities to make new posterity at every age. The administrators are utilized to modify the hereditary creation of people amid portrayal. Fundamentally, the administrators play out an irregular hunt, and can't ensure to yield an enhanced posterity. There are three normal Genetic administrators: crossover, mutation and selection.

\subsection{Selection: -}

One of the genetic algorithm phase is Section; in which browsed singular genomes are later crossover population.

A Selection method which is general might execute like below:

1. The function of fitness is assessed to every person, giving esteems of Fitness; normalized. separating the every individual Fitness estimation of with whole fitness values, with the goal Fitness esteems aggregate which are subsequent measures up to 1 implies Normalization

2. The Fitness standards sliding arranges the Population

3. Accumulate fitness esteems which is normalized figured. The accumulate last individual Fitness ought to obviously 1

4. A R which is Random number in the vicinity of $0 \& 1$ is picked.

5. The individual which selected is entire first that accumulate value which is normalized more noteworthy compared to $\mathrm{R}$.

6. When practice rehashed till sufficient individuals selected, this determination is known Roulette-wheel choice.
Amid the previous 2 decades, Selection techniques various proposed, analyzed, Aas well as looked at. Selection of Regular strategies based the following-

- Selection of the Roulette wheel

- Selection of the Tournament

- Sharing

- Selection of the Truncation

- Selection of Elitist

- Ranking \& scaling

The fitness proportionate selection also known like Roulette wheel selection, which operator of the genetic that chooses possibly helpful Solutions to recombine (Goldberg, 1989). useful for implemented approach. In spite of the fact that wheel selection roulette is exceptionally basic as well as capable, Operator mayn't ensure the chromosomes of unrivalled can be survived as well as passed their own Genes over people to come . Normally, Elitism, methodology which duplicates some current population superior of chromosomes to population, which we are following can be utilized for taking care issue.on the other hand, to use this approach, user can choose the elitism suitable rate. When extremely elitism low rate apply, GA can be misused extra time to detect the standards that are optimal. When elitism high rate connected, GA can be effectively conquer local optimization. In this manner, elitism proper rate Selecting required. Be that as it may, we pick the heuristic crossover as opposed to utilizing Elitism implemented approach, since heuristic hybrid additionally gives more prominent probability which predominant chromosomes attributes protected with one parent chromosome passing without control. The heuristic crossover interest points are clarified in the other ection.

\subsection{Crossover}

One of the fundamental Genetic Operator is Crossover. It worked over 2 Chromosomeson givenoffspring moment\& creates with consolidating the highlights of the two chromosomes. One straightforward method for crossover accomplishing is pick an cut-point of irregular and offspring creationwith consolidating One parent portion one cut point side of other parent fragment to cut-point one side. This technique functions admirably by using bit string portrayal. The GA execution based, all things considered, on the crossover operator execution utilized. The probability of crossover (indicated by PC) specified like quantity of offspring probability that delivers in every age to the Size of population (normally meant with pop size). It regulates the PC normal number $\times$ chromosomes Pop Size for experiencing the operation of crossover. A crossover probability which is higher permits greater amount investigation of arrangement place \& lessens the making odds due with un ideal; however in the event that this high probability, it brings about the considerable wastage calculation time measure in investigating arrangement space unpromising districts . Till today, two or three Crossover executives implemented to encode real numbers, large be taken in to account four classes: conventional, arithmetical, direction-based and stochastic. The administrators which are conventional built with administrators grow to Binary portrayal to the case of Real-Coding. The operators of crood over which are conventional may comprehensively separated with crossover 2 sorts :

- Crossover of Simple: cut point - 1, cut point - 2, multi or uniform cutpoint

- Crossover of Random: crossover of flat, crossover of blend 


\subsubsection{Heuristic Crossover}

crossover of the Heuristic is a standout amongst all well-known crossovers which are real-valued. the operator utilizes the two parent chromosomes fitness estimations to decide the chromosomes of two offspring estimations. Crossover Operator utilization is the one parent Chromosome where Fitness superior to anything other, disregarded forn cutting edge with no control. Just a single chromosome offspring is recently made. operator makes chromosomes as two parent demonstrated as follows: When parent Chromosome Fitness superior to parent fitness..

If the Fitness of the Parent1 Chromosome is superior to the Fitness of the Parent 2 .

$$
\begin{aligned}
& \text { offspring } 1=\text { parent } 1 \\
& \text { offspring } 2=\text { parent } 1 \pm r *(\text { parent } 1-\text { parent } 2)
\end{aligned}
$$

Where $r$ is a Random Incentive in the vicinity of 0 and 1 . The estimation of $r$ is Randomly chosen before every operation of Crossover. Fundamental idea of Heuristic Crossover appearedin 7.

\subsection{Mutation}

One type of foundation operator is the Transformation that produces irregular changes of unconstrained in various Chromosomes. For accomplishing the mutation, one forward scheme to modify minimum one gene. In GA, critical part of the mutation served either (a) supplanting the population gens lost amid the procedure of Selection Therefore attempted the another specific circumstance or (b) giving the absent genes in underlying population. probability of mutation characterized like genes aggregate number level Population. The probability controlled by mutation probability with new qualities brought trail population Till today, a few Mutation administrators implemented for encoding of real numbers.

Mutation Operators which are Arbitrary, for example, equal mutation, mutation of the boundary, and mutation of plain, have a place with the ordinary change administrators, which essentially supplant a predefined extend gene. mutation which is Dynamic (Non uniform mutation) is intended to tweak abilities went for accomplishing high exactness, which is delegated the Arithmetical Mutation Operator.

In this parent chromosome estimation is changed as restricted range thinking about current generation quantity . At point when the current generation count is little, the chromosome changing scope is vast.. The non-uniform change condition of based on the following:

$$
\text { offspring }_{i}=\text { parent }_{i}+\left(\text { upperbound }_{i}-\text { parent }_{i}\right) * f(G)
$$

where $f(G)$ is the range function considering $G$, which is the numberof the current generation. The function $f(G)$ is as follows:

$$
f(G)=\left(r *\left(1-\frac{G}{G_{\max }}\right)^{b}\right.
$$

$G_{\max }$ - Generation's maximum number

b - Parameter of shape

The separation i, A distinction in Fitness esteems among the chromosome which is mutated \& present one contracted with generation count in light of the fact that non-uniform mutation has been planned in light of the Chromosome developed for the ideal esteem when generations increases. Notwithstanding, condition additionally takes the chromosome diversity with Random number $r$ utilizing in meantime.

\section{References}

[1] An effective approach to estimating the parameters of software reliability growth models using a real-valued genetic algorithm TaehyounKima, Kwangkyu Lee b, JongmoonBaik.

[2] A genetic algorithm with real-value coding to optimize multimodal continuous functions, M. Bessaou and P. Siarry. 